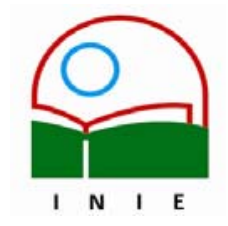

Universidad de Costa Rica

Facultad de Educación

Instituto de Investigación en Educación

ACTUALIDADES INVESTIGATIVAS EN EDUCACION

\title{
EL CONOCIMIENTO PEDAGÓGICO DEL CONTENIDO COMO CATEGORÍA DE ESTUDIO DE LA FORMACIÓN DOCENTE
}

\begin{abstract}
Susan Francis Salazar ${ }^{1}$
Resumen: El análisis de la investigación educativa en Latinoamérica ha mostrado un incremento en el estudio del acto pedagógico, desde su relación con los sujetos que le construyen. Uno de los programas de investigación que hace un significativo aporte a esta línea de estudios es el del "Desarrollo del conocimiento en la enseñanza", auspiciado por la Universidad de Stanford y la Fundación Carnigie, y dirigido por Lee S. Shulman. Este programa ha propuesto al menos cuatro categorías de conocimiento que son base en la toma de decisiones del docente en su hacer pedagógico. Entre ellas se encuentra el "conocimiento pedagógico del contenido". Este artículo se propone revisar el desarrollo conceptual de esta categoría del conocimiento docente y las posibles implicaciones en el estudio de la docencia.
\end{abstract}

Palabras clave: PEDAGOGÍA/ CONOCIMIENTO PEDAGÓGICO DEL CONTENIDO/ ENSEÑANZA/ FORMACIÓN DOCENTE/

Abstract: The analysis of educational research in Latin-American have shown a growing in studies about pedagogical act since the people that build the educational action. One of the research program which make a significant contribution to this field is Knowledge Growing in Teaching sponsored by the University of Stanford and Carnegie Foundation and leaded for Lee S. Shulman. This program proposed at least four knowledge categories for making decisions in teaching, among these categories is the Pedagogical Content Knowledge. This article presents a review about the conceptual development of this category and its implications in the educational research about teaching.

Keywords: PEDAGOGUE/ PEDAGOGICAL CONTENT KNOWLEDGE/ TEACHING/ PROFESSIONAL DEVELOPMENT FOR TEACHING/

1 Candidata a Doctora del Programa Latinoamericano de
Doctorado en Educación de la Universidad de Costa Rica.
Magíster en Evaluación Educativa, Licenciatura en Ciencias de la
Educación con énfasis en Administración Educativa y Bachiller en
Educación Preescolar, todos los títulos en la Universidad de
Costa Rica. En la actualidad labora para el Departamento de
Docencia Universitaria de la Escuela de Formación Docente,
Investigadora del Instituto de Investigación en Educación, dentro
de sus actividades se encuentran el desarrollo del curso Didáctica
Universitaria para profesores de la Universidad de Costa Rica.

Correo electrónico: suagomez@racsa.co.cr

Artículo recibido: 10 de octubre, 2005

Aprobado: 12 de diciembre, 2005 


\section{Introducción ${ }^{2}$}

El docente y la calidad de la educación son elementos que están íntimamente vinculados. Ninguna reforma de la educación tiene posibilidades de éxito sin la activa participación de los y las docentes y su sentimiento de inclusión en la acción educativa y su proyección. Es dentro de esta afirmación donde el estudio de la formación docente, desde la investigación científica, encuentra un terreno poco visitado. Parece haber consenso, entre quienes analizan el desarrollo de la investigación educativa que esta tiene como tarea pendiente la necesidad de profundizar en la investigación pedagógica y los cambios que se producen en la institución educativa, específicamente, en el aula. La permanencia de formas tradicionales en las prácticas pedagógicas en los diferentes niveles educativos, indica un limitado desarrollo en la investigación sobre los procesos de formación y perfeccionamiento docente (Abraham y Rojas, 1997).

El estudio de los procesos de formación profesional docente, es necesario, sobre todo, si se considera que recientes investigaciones han demostrado que estos procesos se han convertido en un importante predictor del logro y rendimiento de los estudiantes (Stronge, $\left.2002^{3}\right)$.

En 1985 Lee S. Shulman como parte de su discurso presidencial de la AERA (American Educational Research Association), planteó la necesidad de indagar en el paradigma perdido de la investigación educativa: El desarrollo del conocimiento del docente en la enseñanza ${ }^{4}$. Lo establece como el programa perdido por la falta de desarrollo investigativo que se concentre en dilucidar las formas de comprensión cognitiva del contenido de la enseñanza por parte de los profesores y profesoras. Con esto postuló el estudio, no sólo de las formas de comportamiento del docente, sino también de su pensamiento. Toda actividad educativa tiene como respaldo una serie de creencias y teorías implícitas que forman parte del pensamiento del docente y que orientan sus ideas sobre el conocimiento, su enseñanza y sobre cómo se construye este o bien cómo se aprende. El desarrollo del pensamiento del docente surge como producto de las condiciones históricas,

\footnotetext{
${ }^{2}$ Este artículo surge como producto de la depuración teórica-conceptual del proyecto de investigación № 724-05-555 del Instituto de Investigación en Educación: Los saberes del profesor universitario: un encuentro con los y las docentes valorados excelentes por sus estudiantes del cual la autora es investigadora principal.

${ }^{3}$ Stronge cita veintidós reportes de investigación que desde 1971 al 2001 plantean como conclusión que existe evidencia consistente sobre los efectos positivos de los programas de formación docente en el rendimiento escolar.
} 
sociales, culturales, personales y otras que los actores y actoras del proceso educativo han desarrollado.

Alfaro (1996) agrega que los participantes del acto educativo, construyen imágenes y metáforas, con las que les dan sentido a las visiones que guían sus acciones. Esto es particularmente importante, si asumimos que estas creencias y teorías implícitas forman parte del basamento que permite la toma de decisiones pedagógicas que hacen los y las docentes. De acuerdo con Shulman (1987) un docente puede transformar la comprensión, las habilidades de desempeño y valores o actitudes deseadas, en acciones y representaciones pedagógicas. Por ello indica que la docencia se inicia cuando el docente reflexiona en qué es lo que debe ser aprendido y cómo será aprehendido por los estudiantes. Es en estos procesos reflexivos donde las creencias, teorías implícitas y otras formas de pensamiento interactúan con las condiciones contextuales, para configurar las acciones que se cristalizan en el aula.

El programa de investigación del desarrollo del conocimiento del docente en la enseñanza ha aportado cuatro importantes categorías teóricas que pueden orientar la investigación educativa al respecto. Dentro de este ámbito, se propone el estudio de la categoría del Conocimiento pedagógico del contenido ${ }^{5}$. Esta categoría fue propuesta por Lee S. Shulman, a partir de 1986, y con ello, promueve el desarrollo de uno de los programas de investigación educativa más importantes a la fecha.

Shulman (1987) propuso que la persona que se dedica a la docencia tiene un conocimiento base que, al menos, incluye siete categorías: el conocimiento del contenido, de lo pedagógico general, de lo curricular, de lo pedagógico del contenido, de los aprendices y sus características, de los contextos educativos y de los fines educativos. Para 1990 estas categorías son redefinidas por Pamela Grossman ${ }^{6}$ (1990) en cuatro áreas generales: el conocimiento pedagógico general, el conocimiento del contenido, el conocimiento pedagógico del contenido y el conocimiento del contexto. Esta última clasificación ha sido la que ha orientado mayoritariamente el desarrollo de este programa de investigación.

\footnotetext{
${ }^{4}$ Traducción libre de "Knowledge Growth in Teaching", se incorpora "del docente" en la traducción pues es un programa referido a los procesos de pensamiento del docente. Este programa es auspiciado por la Universidad de Stanford y la Fundación Carnigie, Estados Unidos (Shulman, 1986)

${ }^{5}$ Esta denominación es la traducción libre del concepto en inglés Pedagogical Content Knowledge (PCK)

- Pamela Grossman forma parte del equipo de investigadores del programa Knowledge Growth in Teaching de la Universidad de Stanford dirigido por Lee S.Shulman.
} 


\section{Conceptualizando el Conocimiento pedagógico del contenido}

Cuando Shulman introduce el término Conocimiento pedagógico del contenido, entre los años 1986 y 1987, presenta los elementos más significativos de este, en al menos tres de sus publicaciones ${ }^{7}$. De acuerdo con Shulman $(1986,1987)$ el Conocimiento pedagógico del contenido (PCK, por sus siglas en inglés) como categoría de conocimiento, involucra los saberes que le permiten al docente hacer enseñable el contenido e incluye:

...las más poderosas formas de representación [...], analogías, ilustraciones, ejemplos, explicaciones y demostraciones, o sea, las formas de representar y formular la materia para hacerla comprensible a otros [...]además la comprensión de qué hace un aprendizaje de tópico específico fácil o difícil. (Shulman, 1986, p. 9)

EI PCK representa "la amalgama del contenido y la pedagogía dentro de una comprensión de cómo temas particulares, problemas o situaciones son organizadas, representadas, ...adaptadas (...) para la enseñanza" (Shulman, 1987, p. 8). Esta afirmación supone poner especial atención a la forma de definir y comprender el contenido y la pedagogía y cómo estos se vinculan.

Esta relación implica que para poder ejercer la docencia, se requiere "la transformación de lo comprendido" de determinado cuerpo disciplinar. O sea, la capacidad de enseñabilidad de determinado contenido descansa, entre otros, en "[...] el conocimiento profundo, flexible y cualificado del contenido disciplinar, pero además, en la capacidad para generar representaciones y reflexiones poderosas sobre ese conocimiento" (Shulman, 1999, p. xi). El estudio del PCK ofrece la oportunidad de entender cómo los y las docentes llegan a hacer enseñables los contenidos. Esta categoría de conocimiento le permite al docente tener la habilidad de convertir sus comprensiones acerca de un tema, en distintas estrategias de enseñanza que le faciliten el logro de los aprendizajes en sus estudiantes. Esto supone cómo los y las docentes "conocedores de la materia" trascienden y se convierten en "maestros de la materia" (Berliner, 1986).

El PCK ha demostrado ser complejo, no sólo como conjunto de conocimientos y habilidades, sino también en las diversas interpretaciones que de esta categoría existen (Fernández-Balboa \& Stiehl, 1995). Desde su aparición el PCK fue objeto de discusión por la ambigüedad en la cual se citó. Cuando Shulman lo propuso, no hizo alusión a las formas

\footnotetext{
7 Nos referimos aquí al capítulo Paradigmas y programas de investigación en el estudio de la enseñanza, incluído en el Manual de investigación en enseñanza, editado por Merlin C. Wittrock en 1986 y los artículos Aquellos que entienden: Desarrollo del conocimiento en la enseñanza publicado
} 
de operacionalizar el conocimiento y mucho menos, a los elementos que permiten definir el conocimiento pedagógico del contenido. Inicialmente Shulman distinguió solamente dos componentes fundamentales en el PCK: el reconocimiento y la comprensión que tiene un docente de los aprendices y de la enseñanza para representar temas específicos.

La consideración de los estudiantes como aprendices involucra el estudio de las preconcepciones, los conceptos erróneos en la construcción de los conocimientos y las dificultades que pueden presentar los estudiantes para aprender determinados contenidos. La posibilidad de conocer y comprender a los estudiantes le permite, al docente, interpretar las acciones e ideas de estos, de modo que puede organizar su enseñanza de una manera más efectiva, puesto que enfoca sus estrategias pedagógicas hacia una mejor representación del contenido. (Magnusson, Krajcik y Borko, 1999)

En este sentido, Shulman $(1986,1987)$ afirma que el manejo profundo de la disciplina, le facilita al docente anticipar los componentes y relaciones del contenido que pueden presentar problemas para su comprensión. Un buen manejo de la disciplina significa saber que algo es así y comprender el por qué de esta naturaleza, pero además saber bajo qué circunstancias se valida este conocimiento: "Esto será importante en las subsiguientes decisiones pedagógicas que consideren el énfasis curricular" (Shulman, 1986, p. 9)

No obstante, el conocimiento profundo de la disciplina se vuelve infructuoso sino se consideran los puntos de vista acerca del contenido que tienen los estudiantes. La generación y uso de actividades significativas de aprendizaje que, por ejemplo, incluyan demostraciones, metáforas y sus variaciones y alternativas para ampliar la comprensión del contenido, dependen, entre otros, de las características del contenido, del dominio de éste por parte del profesor y su conocimiento de los estudiantes. Estas actividades permitirían a los estudiantes ligar el conocimiento previo con la información actual, para la producción de nuevas ideas y reducir las ideas y conceptos erróneos. Estos mismos saberes del docente, le apoyan para tener fluidez en sus discursos y reconocer e identificar las aplicaciones de la disciplina en la vida de sus estudiantes. (Gess-Newsome, 1999)

Diversos estudios han demostrado la necesidad de esta relación entre el conocimiento profundo de la disciplina y de las ideas previas de los estudiantes ${ }^{8}$. Estas investigaciones han demostrado que los y las docentes, con bajo dominio de conocimiento disciplinar, son

en la revista Educational Researcher en 1986 y el artículo Conocimiento y enseñanza: Bases para una nueva reforma, publicado en la revista Harvard Educational Review en 1987. 
menos conscientes de los conocimientos previos de los estudiantes y por tanto, menos capacitados para identificar los errores de concepto que puedan presentar sus estudiantes.

La relación del manejo significativo del contenido con respecto a la selección de las estrategias de enseñanza, considera las distinciones entre cada disciplina. De ahí las expresiones que, con mayor frecuencia escuchamos sobre la no transparencia de los objetos de estudio y por supuesto, los argumentos de que cada disciplina requiere su propia didáctica. Esto fortalece la idea de que cada disciplina tiene una dimensión pedagógica que no está separada de su desarrollo.

A partir de las relaciones de estos componentes, el PCK asume como base, las conexiones entre el conocimiento sobre pedagogía y el conocimiento de la disciplina que tiene el docente. De acuerdo con Shulman (1987) esta interacción es la que permite la transformación del contenido para su enseñanza. Esto sólo ocurre cuando el docente reflexiona e interpreta críticamente la información pedagógica, disciplinar y del contexto. Shulman denominó este proceso de reflexión e interpretación: Modelo de Razonamiento y Acción Pedagógica (Ver Figura 1). Este modelo se basa en el supuesto del que la docencia inicia desde que se piensa cómo se va actuar en el proceso educativo (planificación). Dicho modelo de carácter cíclico y dinámico, toma como punto de partida la reflexión del acto docente desde las intenciones educativas, la estructura conceptual y las ideas que circundan desde lo interno y externo de la disciplina que se va a enseñar. Con esto último, llama la atención a la necesaria comprensión de las relaciones de las intenciones educativas y del contenido con el contexto. Estas comprensiones permitirán la transformación de los contenidos disciplinares en formas representativas que permitan su enseñanza, su evaluación, su reflexión y nuevas comprensiones para un futuro, con lo cual se inicia de nuevo el ciclo.

\footnotetext{
${ }^{8}$ Ver los estudios de Halim \& Mohd.Meerah, 2002; Gess-Newsome, 1999; Magnusson, Krajcik y Borko, 1999, Hogan, Robinowitz \& Craven, 2003; Halim \& Mohd.Meerah, 2002; Darling- Hammond, 1998; Vadya, 1993.
} 
Figura 1 Modelo de razonamiento y acción Pedagógica según Lee S. Shulman (1987)

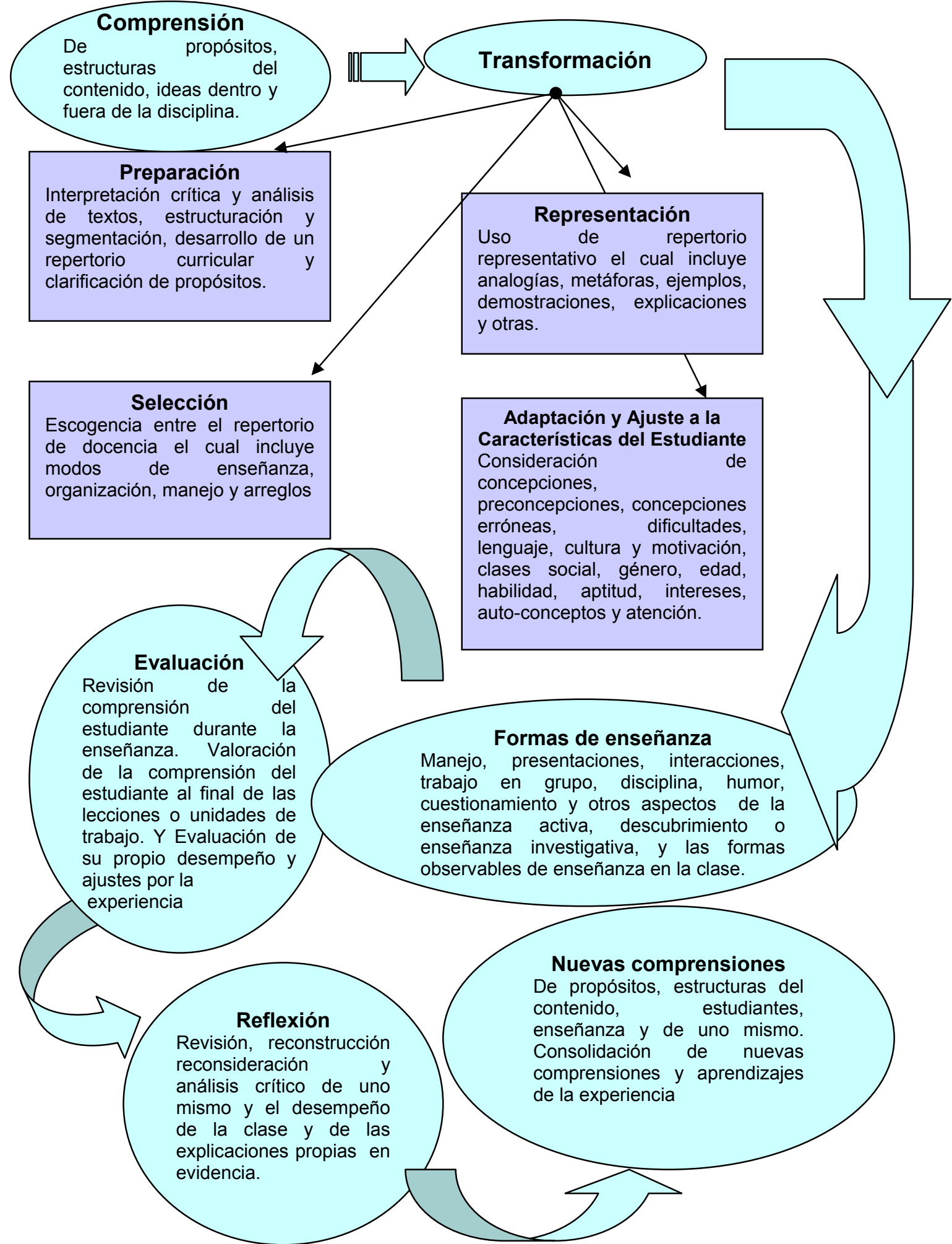

Tomado y adaptado de Shulman (1987, p. 15) 
Por su naturaleza procesal, este modelo de razonamiento y acción pedagógica, muestra procesos de pensamiento del docente en continua reestructuración, sobre el contenido para la enseñanza. Su dinámica se ve enriquecida por el contexto en el cual ocurre, como resultado de las interacciones sociales que el acto educativo envuelve y los distintos momentos que caracterizan el quehacer docente: planeamiento, enseñanza, evaluación, entre otros.

La investigación desarrollada, dentro de este programa, lleva a Grossman (1990) a plantear que los componentes del PCK involucran, además del conocimiento acerca de los estudiantes y de las estrategias didácticas, también del conocimiento del curriculum y del contexto de aprendizaje. El valor del PCK está en la posibilidad que tiene el docente de integrar estos componentes, esta categoría sólo es posible entenderla como un todo, puesto que su carácter transformativo y dinámico, la convierte en una forma de comprensión particular de quienes se dedican a la docencia.

Sin embargo, los procesos que favorecen la formación en el docente, hasta qué punto los planes de estudio de las facultades de educación lo han concebido, como un componente inclusivo de la formación docente, o bien, como elementos fraccionados sin una posibilidad explícita de síntesis, durante el proceso de formación, son áreas poco investigadas.

Grossman (1990) señaló que existen cuatro fuentes desde las cuales es posible observar la generación y desarrollo del PCK: la observación de experiencias de aula, desde su posición como estudiante, hasta como docente en formación; la formación disciplinar, los cursos específicos de pedagogía y la experiencia como docente ya en el aula. Por su parte Gew-Newsome (1999) construyó dos modelos teóricos que explican la formación del PCK: el modelo integrativo y el modelo transformativo. El primero considera el PCK como una categoría producto de la relación de la pedagogía, el contenido y el contexto. El modelo transformativo, por su parte, ve el PCK como el producto de la transformación del conocimiento pedagógico, del contenido y del contexto. (ver Figura 2) 
Figura 2 Modelos del conocimiento docente

Modelo Integrativo

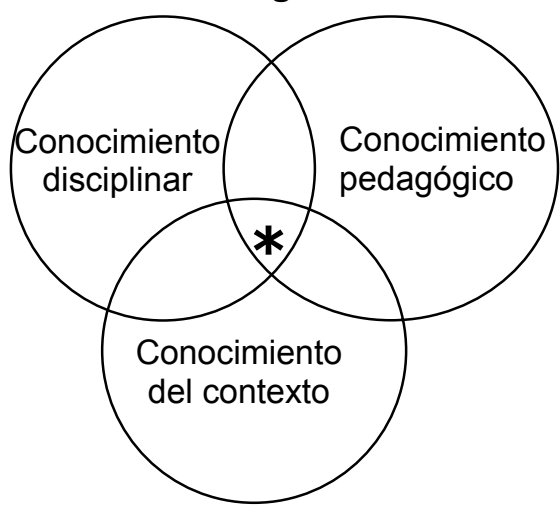

* Conocimiento pedagógico del contenido
Modelo Transformativo

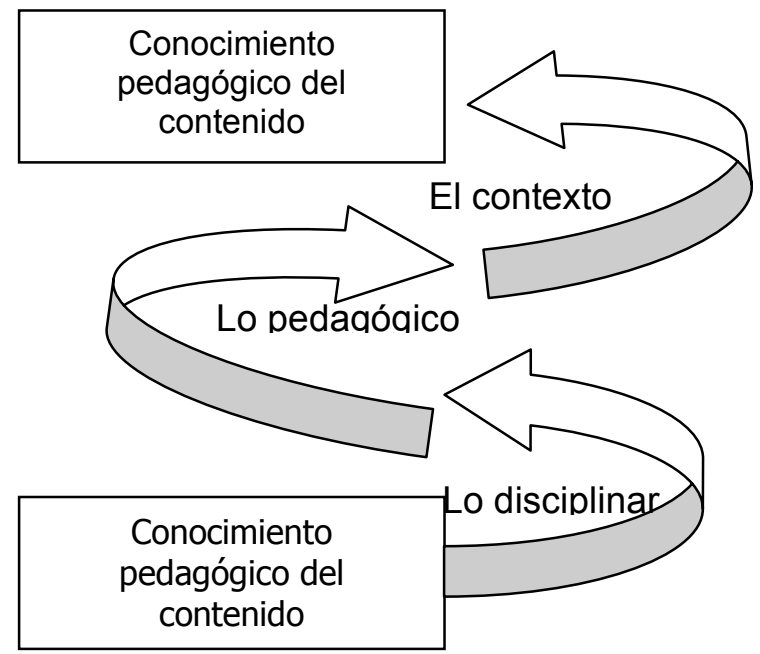

Tomado de Gew-Newsome, J \& Lederman, N. 1999:12

Para este mismo autor, estos modelos representan los extremos de un continuo en el cual el integrativo expresa un marco de conocimiento, donde los saberes disciplinar, pedagógico y del contexto se desarrollan por separado y son integrados en el acto docente. Mientras que el transformativo no se ocupa del desarrollo de estos saberes, sino de cómo son transformados en PCK, como conocimiento base para la docencia. Estos modelos son un importante punto de partida para analizar los planes de formación de docentes.

Actualmente, el desarrollo teórico de esta categoría desarrolla investigación sobre el continuo entre estos dos modelos propuestos por Gew-Newsome. Su impacto se hace evidente en el ámbito de la formación profesional de los y las docentes, tanto en los que están iniciando su proceso formativo como los que ya están en servicio. El debate que promueve recupera una vieja discusión: cuándo y cómo se forma un docente en la pedagogía: cuando ya tiene el conocimiento profundo disciplinar, antes de lo pedagógico, o bien paralelamente, cómo se construye el conocimiento pedagógico del contenido: por partes o al ser producto de las distintas transformaciones que la experiencia le aporta a los y las docentes, cómo puede reconstruirse en los espacios de formación docente. 


\section{Una mirada al PCK desde la conceptualización de lo pedagógico}

El análisis crítico de esta categoría supone retomar la conceptualización de lo pedagógico. Cuando nos referimos a la pedagogía o a sus derivaciones (i.e pedagógico, pedagógica, pedagogismo, entre otros) es común enfrentarse a un debate de carácter difuso, no sólo en su naturaleza, sino también en su génesis. La pedagogía ha sido un concepto que inclusive sirve de "comodín" para describir distintos ámbitos de acción y pensamiento. Es común advertir dentro del campo semántico de la pedagogía términos como ciencia de la educación, práctica educativa, arte de la educación. Además significaciones asociadas con recursos, métodos, prácticas, relaciones, denotaciones relativas al discurso acerca de la enseñanza, del aprendizaje, de la didáctica y connotaciones relativas a la reflexión crítica, a la emancipación y a la liberación individual y social. También se la ha denominado como filosofía de la educación, metodología usada en las acciones de educar y de enseñar, ciencia explicativa de la práctica educativa, entre otros. (Venegas, 1995; Gallego, 1992; Florez, 2000)

Si bien el análisis etimológico de la palabra pedagogía aporta luces sobre la naturaleza y su proyección semántica, también se advierte ambigüedad en esta última. Se mantiene un sentido de "conducción física y espacial" por el origen griego de la palabra". Sin embargo, la misma literatura griega de esa época provee elementos para considerar que su interpretación trasciende este sentido y atribuye un significado de conducción "hacia la humanidad" (García y García, 1996, p. 100)

La pedagogía, tal y como se conoce en la actualidad, tiene su base en el pensamiento científico moderno (siglos XVIII y XIX). Desde ese momento, se origina una cruzada por buscar el carácter científico. Este proceso ha evolucionado paralelamente a la constitución del concepto de ciencia y del desarrollo de metodología para la búsqueda de "verdades": ¿Es la pedagogía ciencia o no lo es? ¿Es la pedagogía la ciencia de la educación o no lo es?

Herbart $^{10}$ propuso formalmente esta cuestión por primera vez y trató de buscar el entramado de ideas que articulara la cientificidad del cuerpo pedagógico. Sin embargo, la dificultad de asumir el carácter de ciencia se circunscribe en la imposibilidad de distinguir, en el desarrollo de la pedagogía, lo científico de lo no científico: "el aspecto metodológico de la

\footnotetext{
${ }^{9}$ Paidogoogía del griego país, paidós, niño, niños, y agoo, agein, conduzco, conducir, designa, en sus orígenes, al esclavo encargado de llevar al niño a la pidea, la escuela. (Gallego - Badillo, 1992).

10 Johann Friedrich Herbart (1776-1841) Se le considera el precursor de la pedagogía científica y hasta de la ciencia psicológica actual. Se constituyó en adversario del idealismo en nombre del realismo filosófico. Su obra clave es Pedagogía general derivada del fin de la educación. (Soto y Bernardini, 1997).
} 
Pedagogía tiene una movilidad y un relativismo intrínseco que no es propio de las adquisiciones científicas" (García y García 1996, p. 111).

La determinación del objeto de estudio de la pedagogía advierte una dificultad más: el conflicto para identificar cuál es este objeto. Si asumimos que el objeto de la pedagogía es la educación, la complejidad estará en determinar sus fronteras con respecto a otras ciencias o saberes. La educación como construcción colectiva es compartida por las ciencias sociales, cuya preocupación es el ser humano como constructor de cultura ${ }^{11}$. Esto explica los aportes desde la sociología, psicología, economía, lingüística, antropología, entre otros, en la misma construcción del discurso pedagógico. Plantear lo educativo desde todos estos nichos teóricos y metodológicos ha derivado en fragmentar la educación como objeto de estudio. Es por esto que se advierte como un reto para la pedagogía determinar su objeto (Florez, 2000).

No se puede omitir que la concepción de ciencia es determinante en la base de esta discusión. Kant en su obra Pedagogía ${ }^{12}$ (2003) planteó la posibilidad de que exista Teoría o Ciencia de la Educación: Pedagogía, siempre que esta última siguiera las vías de la "rigurosidad científica"13 según el pensamiento ilustrado. El pensamiento pedagógico de la época ilustrada representado en Herbart, se propuso la búsqueda de un estatuto científico, una formulación que la identificara como una actividad racional sobre el hecho educativo. Esto obligó a distinguir entre pedagogía y educación.

En la actualidad, dicha discusión permite elaborar algunas conclusiones, entre ellas que lo pedagógico fluye desde el elemento teórico hacia el hecho educativo; describe su ontología, justificación y modelo conceptual de intervención (García y García, 1996). Es una relación dialógica entre las construcciones teóricas y las prácticas educativas, que le permite constituirse históricamente dentro de una colectividad que se conforma de sistemas de representación, de normatividad y de expresión:

\footnotetext{
${ }^{11}$ Compartimos el concepto de cultura de Ros (2001) cuando hablamos de cultura nos referimos tanto a la subjetiva: los significados compartidos, actitudes, normas, valores, que caracterizan a una sociedad, y a la objetiva que se caracteriza por los productos culturales como los sistemas de lengua, tecnología, instituciones políticas, educativas, religiosas entre otras.

${ }^{12}$ El libro "Pedagogía" de Kant aparece por primera vez en 1803 como resultado de los apuntes que recogió Friedrisch Theodor Rink de las clases que el mismo Kant impartió sobre pedagogía. La referencia al 2003 obedece al año de la última edición de esta obra. Ver referencias bibliográficas.

${ }^{13}$ Para García y García (1996) la ciencia para Kant plantea la idea de sistematicidad como la forma de la ciencia: "El postulado, concepto o cadena de conceptos que pueden constituirse en puntos de partida. Posee dos características: estable y originariamente cierto y transmisor de certeza, respecto a algo fuera de sí mismo; las indicaciones y reglas universales de la deducibilidad que se pueden obtener de los postulados es el método, siento ambos correlativos." (p. 115)
} 
Cuando en el discurso pedagógico se alude a la «verdadera educación», tal proposición no tiene otro sentido que el de mera aspiración o la convicción fiducial o ideológica. Indica que las metas educacionales son concordes con el marco simbólico general del contexto cultural, personal o colectivo en cuyo interior fueron formuladas, También indica el nivel de adhesión que a las mismas manifiesta el grupo humano que las mantiene,... (García y García, 1996, p. 180)

Por su carácter antropomórfico la pedagogía alude a procesos educacionales que buscan la constitución humana del hombre y de la mujer, lo que Kant (2003) llamó "humanizar al hombre"14, o sea la búsqueda de valores comportamentales que permiten el desarrollo como ser humano en una sociedad que es histórica y que comparten ideologías y una visión de mundo. Su naturaleza reflexiva se fundamenta en la capacidad de educabilidad del ser humano, de ahí que, se permita prescribir formas de actuación educativas que superan los meros tecnicismos: "Su finalidad es determinar lo que debería ser la educación; o sea, no se orienta hacia el pasado ni hacia el presente, sino hacia el futuro" (Barrantes, 1994, p. 120).

La evolución semántica del concepto de Pedagogía ha permitido llegar a identificar que esta es una ciencia cuyo objeto de estudio es la educación: "la pedagogía es conocimiento, la educación es acción" (García y García, 1996, p. 130). La construcción de una teoría de educación ha implicado la conformación de un campo de conocimientos que explican proposicionalmente el hecho educativo, a partir de la reflexión del pedagogo. No constituye una ciencia formal, puesto que no se inventa su objeto de estudio, más bien es una ciencia fáctica que se refiere a procesos y hechos en la realidad, los cuales para pronunciarlos los observa para confirmar su existencia. Por tanto, comparte el hecho de que todos sus símbolos son interpretaciones que además requieren el encuentro con la realidad: " $L a$ Pedagogía es, por tanto, racional en el sentido de que ha de componerse de conceptos, juicios, raciocinios, no imágenes; el punto de partida y el de llegada son ideas y decisiones de acción" (García y García, 1996, p. 180)

Las decisiones tomadas por el docente para hacer enseñables los contenidos, se traducen en actos pedagógicos. El acto pedagógico se cristaliza en el acto educativo, que por la naturaleza de este último se busca, o sea es intencional. Es ahí cuando el docente

\footnotetext{
${ }^{14}$ La afirmación "humanizar al hombre" responde al proyecto político ilustrado del cual el pensamiento Kantiano forma parte.
} 
concreta el logro de una misión, un objetivo de formación del otro. El acto pedagógico también es un acto consciente. Su comprensión le permite al docente reconocer la forma, en la que él o ella creen, lograran aprender los estudiantes. Es por esto que el acto educativo se reconoce como acto interventivo, como acción práctica y el acto pedagógico, como un acto de reflexión.

El conocimiento pedagógico del contenido tiene implicaciones significativas en la comprensión del papel de los y las educadoras, pues define el abordaje del quehacer docente. Lo que lo hace pedagógico es la búsqueda intencional reflexionada de la transformación intelectual del ser humano en su estructura de conciencia o de saberes (Scheler, citado por Gallego, 1992). Por este motivo, asume la complejidad y diversidad disciplinar que caracteriza la docencia, pues en cada saber existe una transformación intelectual en su estructura conceptual y en sus interrelaciones disciplinares.

Al analizar la relación de lo pedagógico y del contenido, desde el PCK como categoría teórica, es necesario visualizar el valor pedagógico de cada disciplina. Aunque el mismo Shulman establece una diferencia entre el conocimiento pedagógico del contenido y el conocimiento disciplinar, dicha distinción no se justifica epistemológicamente, hablando pues todo conocimiento de una disciplina tiene una dimensión pedagógica. (Segall, 2004; Carlsen, 1999; Mc Evan \& Bull, 1991)

Como ya planteamos, definir "lo pedagógico" se asocia con una búsqueda intencional y reflexionada de la transformación intelectual del ser humano en su estructura de conciencia o de saberes. Como consecuencia, lo pedagógico intenta "influenciar la forma en que los significados son interiorizados, reconocidos, comprendidos, aceptados, confirmados y conectados así como también desafiados distorsionados, superados o falseados" (Simón, citado por Segall, 2004).

Por ello, lo pedagógico no podría ser reducido simplemente a métodos de enseñanza, ni tampoco circunscribir su acción, sólo al espacio del aula. Es posible reconocer lo pedagógico en cualquier ambiente en el cual la reproducción y producción cultural tiene lugar. Según Bernstein (citado Segall, 2004) la pedagogía opera en tres niveles: en la distribución de formas de conciencia, a través de las diferentes formas de conocimiento, en la regulación de la formación de discursos específicos, y en la transmisión de criterios.

Estos tres niveles se pueden observar claramente en diversas prácticas culturales, no necesariamente asociadas al espacio de aula. Un buen ejemplo son las conferencias y encuentros profesionales, en los cuales, se ventilan discursos de producción científica asociados al trabajo profesional, y que exigen la claridad de comunicación, para finalmente 
apoyar la comprensión y enseñabilidad de sus afirmaciones y asegurar que sus representaciones de la realidad encuentran un lugar significativo en las redes de creencias de los otros. (Mc Evan \& Bull, 1991). El conocimiento disciplinar como construcción cultural tiene por tanto, una dimensión pedagógica. La relación del contenido con la pedagogía no es paralela, es intrínseca, inclusiva e inseparable y propone vías de interacción interpersonal, entre los distintos artificios disciplinarios que se crean para su enseñabilidad.

Esto implica que los y las docentes manejan representaciones, culturalmente construidas, no sólo del contenido, sino también de su dimensión pedagógica. Esto nos lleva a otra consideración: el proceso de adquisición de estas representaciones sobre el contenido y su pedagogía. Una representación se puede definir como la construcción mental del mundo, la cual es activamente construida por quien conoce, en este proceso la realidad externa no es independiente del conocedor (Veal \& Kubasko, 2003). La complejidad de estas representaciones van a impactar la capacidad de percibir, inferir, asumir y reconocer situaciones y fenómenos y sus posibles relaciones.

Estas representaciones se construyen en ámbitos culturales conformados de distintas comunidades profesionales y que han desarrollado sus propias formas de ver y comprender el mundo. Sus actividades promueven y justifican su razón de existir, las visiones individuales se constituyen por un acuerdo de consenso y de acciones subjetivas (Veal \& Kubasko, 2003), que van a incluir las decisiones sobre qué, cómo y por qué socializar (incluyendo la enseñanza) determinados tópicos dentro de su disciplina.

Estas representaciones alimentan el proceso de adquisición del PCK. Desde el punto de vista sociohistórico y cultural, el proceso constructivo y de transformación del conocimiento ocurre en contextos sociales, como resultado de las relaciones interpersonales. (Cochran, DeRuiter, King, 1993) En este sentido, Shulman (1986) plantea que las representaciones que forman parte del PCK, son derivadas de la investigación y otras de la práctica. Podemos inferir entonces, que las fuentes de representación se constituyen en las interacciones que las distintas comunidades disciplinares tienen para la producción científica. La reproducción y socialización de estas representaciones, se producen en los planes de formación docente, sobre todo, cuando los y las docentes son formados como especialistas en la enseñanza de determinada disciplina, como ocurre en educación secundaria.

Al interior del desarrollo disciplinar, Shulman indica que la comprensión del contenido requiere la discusión y comprensión de las estructuras de la disciplina, las cuales, además 
difieren según la procedencia del contenido temático. A partir del aporte de Joseph Scwab ${ }^{15}$, Shulman (1986b) distingue dos estructuras en el contenido: la sustantiva y la sintáctica. La estructura sustantiva se refiere a la variedad de formas en las cuales los conceptos y principios básicos de la disciplina están organizados para incorporar sus hechos. Por su parte, la estructura sintáctica es el conjunto de formas en las cuales la verdad y la falsedad, validez o invalidez son establecidas dentro de la disciplina. De nuevo el carácter social de las representaciones culturales se hace presente.

Ambas estructuras van siendo construidas y reafirmadas dentro de las comunidades disciplinares y van guiando su acción profesional y las formas pedagógicas que permiten su mantenimiento, cuestionamiento y reproducción. Esto es determinante en el estudio del PCK, pues el contexto en el cual se aprende y los procedimientos que se utilizan para aprender un conjunto particular de conocimientos y habilidades, llegan a ser una parte fundamental de lo aprendido (Putnam \& Borko, 2000)

Lo anterior supone que las formas epistemológicas como procesos de construcción de conocimiento y que están detrás de los distintos enfoques disciplinares que manejan los y las docentes, han sido establecidas en el interior de sus comunidades disciplinares y orientan su indagación, comunicación así como sus prácticas profesionales y pedagógicas y, por ello, las formas de representación que utilizan para enseñar determinados contenidos se ven permeadas por los valores definidos por la parte sustantiva de la disciplina y por la parte sintáctica.

\section{Algunas consideraciones finales}

El recorrido conceptual del PCK permite comprender la necesidad de revisar, analizar e investigar los planes de formación docente desde esta categoría, sin olvidar que, existe una particularidad disciplinar que recorre los contenidos y su dimensión pedagógica. Es importante también tomar en cuenta que los y las docentes en formación, han construido representaciones sobre los procesos pedagógicos, no sólo como producto del proceso formal profesional de preparación para la docencia, sino también desde su experiencia de estudiante en los niveles educativos anteriores y su vinculación, a partir de las distintas redes sociales a su alrededor, con otras manifestaciones disciplinares.

\footnotetext{
15 Joseph Schwab (1978) es citado por Lee Shulman en su artículo Those who Understand (1996). Shulman toma como referencia los ensayos de Schwab sobre las formas de representar el contenido disciplinar, en este caso, a partir de las estructuras sustantivas y sintáctica.
} 
Esta reflexión es urgente y necesaria a la luz de la responsabilidad del docente en los procesos formativos iniciales en nuestra sociedad. La carrera por marcar responsables en el desempeño de lo educativo viene observando de cerca de los y las docentes, y por ende, también a los y las responsables de su formación, en nuestro país, las universidades. No podemos obviar que el carácter científico que nos propone la pedagogía nos obliga a transformar las acciones educativas orientadas a la formación docente, no es solamente conocer técnicas didácticas, entre otros, lo que da autoridad para el desarrollo de procesos educativos.

El carácter científico de la pedagogía clama por procesos transformadores en la formación docente, que basados en procesos serios de investigación, permita el desarrollo de docentes capaces de reflexionar y comprender las relaciones de las intenciones educativas con el contenido y con el contexto, que por tanto, se convierta en un investigador crítico y analítico de su práctica y de la práctica en su contexto. Un docente capaz de reconocer como la estructura disciplinar constituye una amalgama de creencias, valores y actitudes que acompañan los distintos relatos que han construido para comprender y explicar las verdades de nuestra vida a los otros.

La investigación sobre esta categoría teórica puede revelar áreas ocultas que no se han considerado en la formación docente y que pueden estar impactando, de forma significativa, el desempeño de los y las docentes en el sistema educativo nacional. Esta categoría brinda un ámbito importante de reflexión sobre los conceptos relacionados con la enseñabilidad de los contenidos y la capacidad de los y las docentes para crear formas de representación que permitan a los estudiantes construir conocimiento. También aporta un importante espacio para discutir la profundidad de conocimiento disciplinar que requieren los y las profesores para poder generar formatos didácticos, a partir de su comprensión.

La profundización en los estudios al respecto, podrían llegar a develar caminos hacia una formación docente con mayor grado de contextualidad y comprensión disciplinar y por tanto, pedagógica, sin caer en modelos que han provocado más un activismo didáctico y no la búsqueda de construcciones de conocimiento por parte de los estudiantes.

\section{Referencias}

Abraham, N.M. \& Rojas, F.A. (1997). La investigación educativa en Iberoamérica. Revista de Educación. 312: 21-41. 
Alfaro,V.G. (1996). Evaluación cualitativa: Técnicas y estrategias. En Tendencias actuales en la medición y evaluación educativa. Compiladora Leda Badilla Chavarría San José: Universidad de Costa Rica.

Barrantes, R. (1994). Pedagogía y educación. Acta Académica. 14: 116-121

Berliner, D.C. (1986). In pursuit of the expert pedagogue. Educational Research. 15(7): 513.

Carlsen, W.S. (1999). Domains of teacher knowledge, in J. Gess-Newsome, and N.G. Lederman, (Eds.). Examining Pedagogical Content Knowledge: The Construct and its Implications for Science Teaching (pp. 133-147). Dordrecht: Kluwer.

Cochran, K., De Ruiter, J. \& King, R. (1993). Pedagogical Content Knowing: An integrative model for Teacher Preparation. Journal of Teacher Education. 44(4): 263-273.

Darling-Hammond, L. (1998). Teacher Learning that Supports Students Learning. Educational Leadership. 55(5): 6-12.

Fernández-Balboa, J. \& Stielkl. (1995). The Generic Nature of Pedagogical Content Knowledge among College Professor. Teaching and Teacher Education. 11 (3): 293306.

Flórez, O. R. (1995). Hacia una pedagogía del conocimiento. Colombia: McGraw-Hill Interaméricana.

Gallego-Badillo, R. (1992). Saber Pedagógico: Una visión alternativa. Santa Fe de Bogotá: Cooperativa Editorial Magisterio.

García, C. J. y García del Dujo, A. (1996). Teoría de educación. España: Santillana.

Gess-Newsome, J. (1999). Knowledge and Beliefs about Subject Matter. In J.GessNewsome and N.G. Lederman (Eds), Examining Pedagogical Content Knowledge: The Construct and its Implications for Science Teaching (pp. 51-95). Dordrecht: Kluwer.

Grossman, P.L. (1990). The making of a teacher: Teacher knowledge and teacher education. New York: Teachers College Press.

Halim, L. \& Mohd.Meerah, S. (2002). Science Trainee Teachers' Pedagogical Content Knowledge and its Influence on Physics. Teaching Research in Science \& Technological Education. 20(2): 215-225

Hogan, T. \& Rabinowitz, M. (2003). Representation in Teaching: Inferences from Research of Expert and Novice. Teachers Educational Psychologist. 38(4): 235-247

Kant, I. (2003). Pedagogía. 3a. ed. Madrid España: Ediciones Akal.

Magnusson, Krajcik y Borko. (1999). Nature, Sources, and Development of PCK. In J.GessNewsome and N.G. Lederman (Eds), Examining Pedagogical Content Knowledge: 
The Construct and its Implications for Science Teaching (pp. 95-132). Dordrecht: Kluwer.

Mc Ewan, H\& Bull, B. (1991). The pedagogic nature of subject matter knowledge. American Educational Research Journal. 28: 316-334

Putnam, R.T. \& Borko, H. (2000). What Do New Views of Knowledge and Thinking Have to Say About Research on Teaching Learning. Educational Researcher. 29(1): 4-15.

Ros, M. (2002). Los valores culturales y el desarrollo socioeconómico: Una comparación entre teorías culturales. REIS 99: 9-33

Schwab, J.J. (1978). Science, curriculum and liberal education. Chicago: University of Chicago Press.

Segall, A. (2004). Revisiting Pedagogical Content Knowledge: The Pedagogy of Content/the Content of Pedagogy. Teaching and Teacher Education. 20(5): 489-504.

Shulman, L. (1986). Those who understand: Knowledge growth in teaching. Educational Researcher. 15(2): 4-14.

Shulman, L. (1999). Forward. In J.Gess-Newsome and N.G. Lederman (Eds), Examining Pedagogical Content Knowledge: The Construct and its Implications for Science Teaching (pp. ix-ii). Dordrecht: Kluwer.

Shulman, L. Wittrock, M (Ed). (1987). Paradigmas y programas de investigación en el estudio de la enseñanza: Una perspectiva contemporánea La investigación de la enseñanza. Barcelona: Editorial Paidós

Shulman, L.S. (1987). Knowledge and Teaching: Foundations of the new reform. Harvard Educational Review. 57: 1-22

Soto, J.A. y Bernardini, A. (1997). La educación actual en sus fuentes filosóficas. San José, Costa Rica: Editorial Universidad Estatal a Distancia.

Stronge, J.H. (2002). Qualities of Effective Teachers. Alexandría,Va. EE.UU.: Association for Supervision and Curriculm Development.

Vaidy, S.R. (1993). Restructuring Elementary and Middle School Science for Improved. Teaching and Learning Education. 114(1): 62-71.

Veal, W. \& Kubasko, D. (2003). Biology and Geology Teachers' Domain-Specific Pedagogical Content Knowledge of Evolution. Journal of Curriculum and Supervision. 18(4): 334-352.

Venegas R. M. (1995). La función docente en la Universidad de Costa Rica. El caso de las y los y las docentes de las áreas de Ciencias Básicas y Ciencias Sociales. Tesis de maestría. Universidad de Costa Rica, Costa Rica. 